\title{
Methods in Molecular Biology
}

Series Editor

John M. Walker

School of Life Sciences

University of Hertfordshire

Hatfield, Hertfordshire, AL10 9AB, UK

For further volumes:

http://www.springer.com/series/7651 



\title{
Bioinformatics and Drug Discovery
}

\section{Second Edition}

\author{
Edited by
}

\section{Richard S. Larson}

The University of New Mexico, Albuqerque, NM, USA

漶 Humana Press 


\section{Editor}

Richard S. Larson

The University of New

Mexico, Albuqerque

NM, USA

ISSN 1064-3745

ISBN 978-1-61779-964-8

DOI 10.1007/978-1-61779-965-5

Springer New York Heidelberg Dordrecht London
ISSN 1940-6029 (electronic) ISBN 978-1-61779-965-5 (eBook)

Library of Congress Control Number: 2012940934

\section{(C) Springer Science+Business Media New York 2012}

This work is subject to copyright. All rights are reserved by the Publisher, whether the whole or part of the material is concerned, specifically the rights of translation, reprinting, reuse of illustrations, recitation, broadcasting, reproduction on microfilms or in any other physical way, and transmission or information storage and retrieval, electronic adaptation, computer software, or by similar or dissimilar methodology now known or hereafter developed. Exempted from this legal reservation are brief excerpts in connection with reviews or scholarly analysis or material supplied specifically for the purpose of being entered and executed on a computer system, for exclusive use by the purchaser of the work. Duplication of this publication or parts thereof is permitted only under the provisions of the Copyright Law of the Publisher's location, in its current version, and permission for use must always be obtained from Springer. Permissions for use may be obtained through RightsLink at theCopyright Clearance Center. Violations are liable to prosecution under the respective Copyright Law.

The use of general descriptive names, registered names, trademarks, service marks, etc. in this publication does not imply, even in the absence of a specific statement, that such names are exempt from the relevant protective laws and regulations and therefore free for general use.

While the advice and information in this book are believed to be true and accurate at the date of publication, neither the authors nor the editors nor the publisher can accept any legal responsibility for any errors or omissions that may be made. The publisher makes no warranty, express or implied, with respect to the material contained herein.

Printed on acid-free paper

Humana Press is a brand of Springer

Springer is part of Springer Science+Business Media (www.springer.com) 


\section{Preface}

Recent advances in drug discovery have been rapid and impressive. When we published the first edition of Bioinformatics and Drug Discovery, our goal was to facilitate employment of these new technologies in the process of drug discovery. We detailed a pipeline of methods and techniques that could be used. In just a few short years, these technologies have become much more powerful and robust. To address this challenge, this edition has been completely updated to include topics that range from new technologies in target identification, to genomic analysis, cheminformatics, protein analysis, and network or pathway analysis. In addition, it is nearly impossible for an investigator to be familiar with all these techniques. As a result, we have formatted our chapters slightly differently than most published by Humana Press. Each chapter provides an extended introduction that describes the theory and application of the technology. In the second part of each chapter, we have incorporated detailed procedures related to the use of these technologies and software. Even the pipeline of methodologies and the biologic analysis that they perform have grown and evolved.

Bioinformatics and Drug Discovery targets those interested in the different aspects of drug design, which include academicians (biologists, informatists, chemists, and biochemists), clinicians, and scientists at pharmaceutical companies. All the chapters in this edition of Bionformatics and Drug Discovery have been written by well-established investigators who use the methods on a regular basis. In all, this book is designed to provide readers not only with the planned insightful overview of key topics but also with the customary ample supply of unfailing reproducible step-by-step procedures for techniques described. 



\section{Acknowledgments}

I wish to acknowledge the significant contribution of Ms. Emily Morelli for her administrative organization of contributors' materials and her continual communication with the contributing authors. 



\section{Contents}

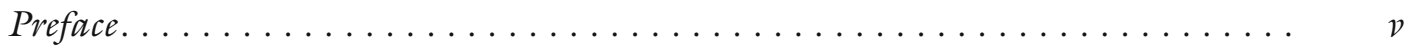

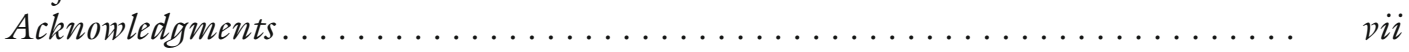

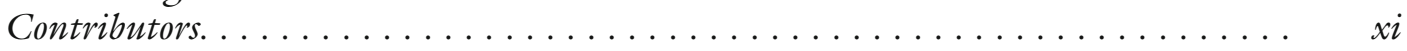

1 Cell Perturbation Screens for Target Identification by RNAi . . . . . . . . . $\quad 1$ Kubilay Demir and Michael Boutros

2 Using Functional Genomics to Identify Drug Targets: A Dupuytren’s

Disease Example . . . . . . . . . . . . . . . . . . . . . . 15

Mivela Sedic, Sandra Kraljevic Pavelic, and Karlo Hock

3 Functional Characterization of Human Genes from Exon Expression and RNA Interference Results. . . . . . . . . . . . . . . . . 33

Dorothea Emig, Hagen Blankenburg, Fidel Ramivez, and Mario Albrecht

4 Barcode Sequencing for Understanding Drug-gene Interactions . . . . . . . . . .

Andrew M. Smith, Tanja Durbic, Saranya Kittanakom, Guri Giaever, and Corey Nislow

5 High-Throughput Sequencing of the Methylome Using Two-Base Encoding . Christina A. Bormann Chung

6 Applications and Limitations of In Silico Models in Drug Discovery . . . . . . . Abmet Sacan, Sean Ekins, and Sandbya Kortagere

7 Compound Collection Preparation for Virtual Screening. . . . . . . . . . . . .

Cristian G. Bologa and Tudor I. Oprea

8 Mapping Between Databases of Compounds and Protein Targets . . . . . . . 145 Sorel Muresan, Markus Sitzmann, and Christopher Southan

9 Predictive Cheminformatics in Drug Discovery: Statistical Modeling for Analysis of Micro-array and Gene Expression Data. . . . . . . . . . . . . 165 N. Sukumar, Michael P. Krein, and Mark J. Embrechts

10 Advances in Nuclear Magnetic Resonance for Drug Discovery. . . . . . . . . . 195 Laurel O. Sillerud and Richard S. Larson

11 Human ABC Transporter ABCG2 in Cancer Chemotherapy:

Drug Molecular Design to Circumvent Multidrug Resistance

Toshibisa Ishikawa, Hikaru Saito, Hiroyuki Hirano, rutaka Inoue, and roji Ikegami

12 Protein Interactions: Mapping Interactome Networks to Support

Drug Target Discovery and Selection

Javier De Las Rivas and Carlos Prieto 
13 Linking Variants from Genome-Wide Association Analysis to Function via Transcriptional Network Analysis . . . . . . . . . . . . . . . . . 297 Benjamin J. Keller, Sebastian Martini, and Viji Nair

14 Models of Excitation-Contraction Coupling in Cardiac Ventricular Myocytes . 309 M. Saleet Jafri

15 Integration of Multiple Ubiquitin Signals in Proteasome Regulation . . . . . . 337 Marta Isasa, Alice Zuin, and Bernat Crosas

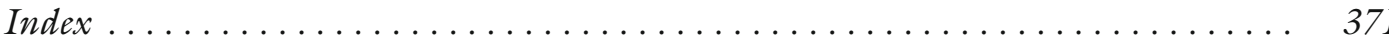




\section{Contributors}

Mario Albrecht • Max Planck Institute for Informatics, Saarbrïcken, Germany

Hagen Blankenburg - Max Planck Institute for Informatics, Saarbrücken, Germany

Cristian G. Bologa - Department of Biochemistry and Molecular Biology,

University of New Mexico School of Medicine, Albuquerque, NM, USA

Michael Boutros - Division of Signaling and Functional Genomics,

Department for Cell and Molecular Biology, German Cancer Research Center (DKFZ),

Heidelberg University, Heidelberg, Germany

Christina A. Bormann Chung • Life Technologies, Foster City, CA, USA

Bernat Crosas - Proteasome Regulation Lab, Cell Biology Department,

Institute of Molecular Biology of Barcelona (CSIC), Barcelona, Spain

Kubilay Demir • Division of Signaling and Functional Genomics,

Department for Cell and Molecular Biology, German Cancer Research Center (DKFZ),

Heidelberg University, Heidelberg, Germany

Tanja Durbic - Donnelly Centre, University of Toronto, Toronto, ON, Canada

SEAN EKIns - Collaborations in Chemistry, Jenkintown, PA, USA; Department of Pharmacentical Sciences, University of Maryland, MD, USA;

Department of Pharmacology, University of Medicine and Dentistry of New Jersey

(UMDNJ)-Robert Wood Johnson Medical School, Piscataway, NJ, USA

MARK J. Embrechts • Department of Industrial and Systems Engineering,

Rensselaer Polytechnic Institute, Troy, NY, USA

Dorothea Emig - Max Planck Institute for Informatics, Saarbrücken, Germany

Guri Giaever - Donnelly Centre, University of Toronto, Toronto, ON, Canada

Hiroyuki Hirano • Graduate School of Bioscience and Biotechnology,

Tokyo Institute of Technology, Yokohama, Japan

Karlo Hock - Department of Ecology, Evolution and Natural Resources,

Rutgers The State University of New Jersey, New Brunswick, NJ, USA

Yoji Ikegami - Department of Drug Metabolism and Disposition,

Meiji Pharmaceutical University, Tokyo, Japan

Yutaka Inoue • Department of Drug Metabolism and Disposition,

Meiji Pharmaceutical University, Tokyo, Japan

Marta Isasa - Proteasome Regulation Lab, Cell Biology Department,

Institute of Molecular Biology of Barcelona (CSIC), Barcelona, Spain

ToshiHisa IsHikawa - Graduate School of Bioscience and Biotechnology,

Tokyo Institute of Technology, Yokohama, Japan

M. SAlEet JAFri - School of Systems Biology, George Mason University, Manassas, VA, USA

Benjamin J. Keller • Department of Computer Science, Eastern Michigan University,

Ypsilanti, MI, USA

Saranya KitTanakom - Donnelly Centre, University of Toronto, Toronto, ON, Canada

SANDhya Kortagere - Department of Microbiology and Immunology,

Drexel University College of Medicine, Philadelphia, PA, USA 
Michael P. Krein • Rensselaer Exploratory Center for Cheminformatics Research and Department of Chemistry and Chemical Biology, Rensselaer Polytechnic Institute, Troy, NY, USA

Richard S. Larson - The University of New Mexico, Albuqerque, NM, USA

Sebastian Martini • Nephrology Division, Department of Internal Medicine, University of Michigan, Ann Arbor, MI, USA

Sorel Muresan • DECS Global Compound Sciences, Computational Chemistry, AstraZeneca RéD, Mölndal, Sweden

VIJI NAIR • Nephrology Division, Department of Internal Medicine, University of Michigan, Ann Arbor, MI, USA

Corey Nislow • Donnelly Centre, University of Toronto, Toronto, ON, Canada

Tudor I. Oprea - Department of Internal Medicine, Translational Informatics Division, University of New Mexico, Albuquerque, New Mexico

Sandra Kraljevic Pavelic - Department of Biotechnology, University of Rijeka, Rijeka, Croatia

Carlos Prieto • Biotechnology Institute of Leon (INBIOTEC), Leon, Spain

Fidel Ramírez • Max Planck Institute for Informatics, Saarbrücken, Germany

Javier De Las Rivas - Bioinformatics and Functional Genomics Group,

Cancer Research Center (IBMCC, CSIC/USAL), Salamanca, Spain

Ahmet Sacan • School of Biomedical Engineering, Drexel University, Philadelphia, PA, USA

HiKarU SAITO - Graduate School of Bioscience and Biotechnology, Tokyo Institute of Technology, Yokohama, Japan; Oral Formulation Development, Pharmaceutical Research and Technology Laboratories, Astellas Pharma Inc, Shizuoka, Japan

Mirela SEDiC - Department of Biotechnology, University of Rijeka, Rijeka, Croatia

Laurel O. Sillerud - Department of Biochemistry and Molecular Biology UNM HDC, University of New Mexico, Albuquerque, NM, USA

Markus Sitzmann - Chemical Biology Laboratory, Center for Cancer Research, National Cancer Institute, National Institutes of Health, Frederick, MD, USA

Andrew M. Smith • Donnelly Centre, University of Toronto, Toronto, ON, Canada

Christopher Southan • DECS Global Compound Sciences, Computational Chemistry, AstraZeneca RéD, Mölndal, Sweden; ChrisDS Consulting, Göteborg, Sweden

N. Sukumar - Rensselaer Exploratory Center for Cheminformatics Research and Department of Chemistry and Chemical Biology, Rensselaer Polytechnic Institute, Troy, NY, USA

Alice Zuin - Proteasome Regulation Lab, Cell Biology Department, Institute of Molecular Biology of Barcelona (CSIC), Barcelona, Spain 